Voix et Images

volxetimages

\title{
Essayistes du cru dans un terreau profond
}

\section{Robert Major}

Volume 18, numéro 1 (52), automne 1992

Les écritures masculines

URI : https://id.erudit.org/iderudit/201005ar

DOI : https://doi.org/10.7202/201005ar

Aller au sommaire du numéro

Éditeur(s)

Université du Québec à Montréal

ISSN

0318-9201 (imprimé)

1705-933X (numérique)

Découvrir la revue

Citer cet article

Major, R. (1992). Essayistes du cru dans un terreau profond. Voix et Images, 18(1), 144-152. https://doi.org/10.7202/201005ar d'utilisation que vous pouvez consulter en ligne.

https://apropos.erudit.org/fr/usagers/politique-dutilisation/ 


\section{Essayistes du cru dans un terreau profond}

\section{Robert Major, Université d'Ottawa}

Il y a trente ans, L'Hexagone s'était taillé la réputation d'être la maison par excellence de la poésie québécoise. À juste titre. Créée par des poètes, elle rassemblait les meilleurs d'entre eux et multipliait les publications importantes, au moment, d'ailleurs, où la poésie était le moyen d'expression privilégié des écrivains du Québec. Les recueils de L'Hexagone, inédits ou rétrospectives, ceux des Gaston Miron, Michel van Schendel, Jean-Guy Pilon, Fernand Ouellette, Rina Lasnier, Yves Préfontaine, Alain Grandbois, Roland Giguère, Gilles Hénault, Paul-Marie Lapointe, sont ainsi devenus autant de repères essentiels dans notre aventure littéraire.

Ces derniers temps, c'est avec d'autres recueils, d'un autre genre, que L'Hexagone s'impose. J'ai sous la main les treizième et quatorzième volumes de la collection - Essais littéraires ", collection dont les titres et le rythme de production forcent l'attention. Ma dernière chronique rendait compte des livres d'André Brochu et de Joseph Bonenfant, publiés dans cette collection (onzième et douzième titres). Depuis, Jean Marcel a publié Pensées, passions et proses $^{1}$ et Laurent Mailhot, Ouvrir le livre ${ }^{2}$. Je regrette de n'avoir pu aborder, au même moment, ces qúatre livres importants, recueils de même nature, œuvres de critiques qui, dans des registres différents, avec des préoccupations diverses mais convergentes, ont pu marquer l'évolution de la critique québécoise et notre lecture des œuvres d'ici. Les aléas de la production, sur lesquels un chroniqueur n'a aucune prise, ne l'ont pas voulu. Deux chroniques consécutives sur des livres de même type (reprises d'articles disséminés tout au long d'un parcours d'une vingtaine d'années) et chez le même éditeur: j'ai quelque peu hésité. Mes scrupules furent levés par l'intérêt des textes et l'importance des contributions. Levés aussi par la présence, chez ces deux critiques (comme chez les deux précédents dans cette collection, d'ailleurs), d'une certaine parenté intellectuelle dont la critique québécoise tire le plus grand profit et qu'il n'est pas inutile ou inintéressant de signaler. 
En effet, Jean Marcel et Laurent Mailhot sont de ces critiques (peut-on parler de génération?) qui sont venus à la littérature québécoise après avoir été formés à la française. Laurent Mailhot s'est d'abord fait connaître comme spécialiste d'Albert Camus; Jean Marcel est médiéviste, et même spécialiste des "choses très anciennes", ou des :temps perdus", ces sources lointaines de la littérature française qu'est l'Antiquité. Deux essayistes du cru, donc, mais qui plongent de longues racines tentaculaires dans un terreau profond, qui déborde les limites du trécarré. La lecture de leurs essais nous fait voir le grand avantage pour les études québécoises de cette compétence première dans un autre corpus. Quand le monde est sa province, quelles richesses n'apporte-t-on pas à la compréhension de la littérature de son pays. C'est ce qui s'appelle une culture.

Laurent Mailhot a choisi d'exclure de son recueil tout ce qui ne touchait pas à la littérature québécoise. La littérature française reste présente dans son livre, toutefois, par ses références, ses allusions, ses digressions, ses modèles de lecture. Jean Marcel, quant à lui, a choisi d'inclure des articles qui remontent aux origines. L'effet est le même dans les deux cas. Leurs analyses se fondent sur tout un arrière-plan culturel et elles s'en nourrissent. Et à lire l'un et l'autre critique, l'évidence s'impose. Littérature québécoise, littérature française, littérature mondiale? Cela est tout un. La littérature française est la nôtre et tout ce que la langue et la culture française ont intégré nous appartient en propre. Qu'on se le dise! Jean Marcel, d'ailleurs, le dira, et clairement, dans un essai qui devrait devenir le vade-mecum de tous les intellectuels québécois (ceux qui réfléchissent, et les autres, aussi):

Qu'est-ce donc, en définitive, que la langue française? C'est ce par quoi j'exerce un droit à l'héritage non seulement de tout ce qui a été produit dans cette langue (ce qui n'est déjà pas mal, merci), mais aussi à l'essentiel de l'héritage de l'humanité qui y a été versé par contamination, contact ou traduction. Cela est immense. Cela est un empire qui couvre tous les âges de l'humanité historique et recouvre la quasi-totalité des terres de la planète. Pourquoi m'en priverais-je? Pourquoi me désisteraisje de cet héritage, l'un des plus riches que l'on puisse recevoir?

Trois ou quatre autres langues, tout au plus, dans le monde actuel pourraient m'offrir pareille quantité et pareille qualité de richesses culturelles. Il s'agit là d'un privilège considérable dont nous, Québécois, avons bien peu abusé, me semble-t-il, et dont nous nous sommes à peine prévalus. Quoi donc! nous avons à portée de la main, par ce réseau extraordinaire d'information qu'est la langue, une culture unique dans l'histoire de l'humanité, et nous serions les seuls, semble-t-il, a la dédaigner? Par quelle aberration le Québec serait-il donc le seul pays au monde où se ferait jour une hostilité à l'égard de la culture que sa langue propre met à sa disposition, et généreusement? (p. T) 
Jean Marcel, par la facture même de son livre, nous indique bien qu'il entend profiter du privilège. Il revendique tout. Pas question de tourner le dos à la moindre parcelle de son héritage.

\section{Le désir d'une pensée}

-La quête d'un peu de sens. (p. 9); "le désir d'une pensée. (p. 10); la ssauvage passion de vouloir connaître, de découvrir, de chercher, de rechercher - (p. 10). Ainsi s'exprime Jean Marcel dans sa Présentation, lorsqu'il examine, avec un certain détachement attendri, les textes recueillis pour ce livre, témoins épars de son passé. Et sans doute a-t-il raison de mettre ce recueil sous le signe d'une passion intellectuelle. La ferveur y est, de même que l'intelligence. Il n'est pas fortuit, d'ailleurs, que l'auteur signe ce livre de son demi-pseudonyme. Au-delà de l'anecdote racontée dans la Présentation, la signature indique clairement la volonté d'effacer la ligne de démarcation entre l'écriture savante (universitaire) de certains de ces écrits qui ont été, à l'origine, signés du nom complet (Jean-Marcel Paquette), et l'écriture polémique, artistique, "non spécialiste* des autres. Tous ces textes sont ramenés dans le giron d'un unique auteur, désormais réfractaire aux cloisons, cherchant, dans sa signature unique, à affirmer le souci de sa prose et sa subjectivité.

Certes, il n'est pas dit que la volonté d'effacer les cloisons les fasse nécessairement disparaître. Mais dans l'ensemble ces articles voisinent bien les uns avec les autres. Les intérêts de l'auteur sont fort variés, sa curiosité sans bornes, et ces écrits témoignent d'une intelligence, d'une finesse, d'une clarté d'exposition, d'une culture, aussi (dans le sens d'un savoir, mais aussi d'une profondeur de réflexion et d'une largeur de vues), auxquelles Jean Marcel nous a habitués, certes, mais qui n'en demeurent pas moins remarquables pour autant.

Le livre est divisé en cinq parties bien équilibrées. * De choses très anciennes" nous promène d'Alexandrie (quatrième siècle) au Canada dont Charlevoix est l'historiographe, en passant par le Moyen Âge (Villon et une poétique de la mort) et l'Occident faustien. - Des questions de langue et d'identité * aborde ces sempiternels problèmes du mal-être québécois (langue, culture, éducation, norme, conflits et lois linguistiques), mais réussit néanmoins, avec fougue et intelligence, à y ajouter du nouveau et un peu de clarté. • De la littérature québécoise. présente un survol exemplaire du corpus québécois et des études particulières sur Jacques Ferron, Marie-Claire Blais, Réjean Ducharme, Lionel Groulx, Gustave Lamarche, Pierre Vadeboncœur, Gérard Bessette, ainsi que des aperçus des revues Études littéraires et Liberté. 
- Du cinéma et de l'opéra * peut aussi bien analyser les trois versions cinématographiques de Maria Cbapdelaine que celles de Tristan ou de Lancelot par Lagrange et Bresson, étudier l'opéra Menaud, maîtredraveur de Marc Gagné ou l'immense cycle de Wagner, passer d'une réflexion sur les rapports entre littérature et cinéma à une analyse du Manon Lescaut de Puccini. La dernière section, finalement, (*De l'essai.) reprend un certain nombre d'articles fondamentaux sur la théorie de l'essai ainsi que des analyses de Gilles Leclerc, de Jacques Ferron romancier-essayiste et de la sociocritique de Lucien Goldmann.

Si j'avais à faire un choix dans cette matière abondante, choix arbitraire, et sans doute davantage révélateur de mes préjugés et de mes ignorances que de la qualité intrinsèque des essais, je retiendrais particulièrement les textes suivants, soit qu'ils m'aient le plus appris, soit qu'ils m'aient spécialement plu: „Fragments de lettres à un ami sur les rapports de la langue et la culture ,, pour la rigueur de la réflexion et son mélange remarquable de passion, de sérénité, de désabusement et de foi; " Une poétique humaine de la divinité: Gustave Lamarche", pour la maîtrise déployée dans l'analyse d'un sujet difficile et pour la valorisation d'un poèté marginalisé (que dis-je: ignoré par la critique québécoise moutonne!); * Littérature et cinéma: une histoire d'amourpassion *, pour l'ample survol de la civilisation auquel nous sommes conviés afin de mettre un peu d'ordre dans la question des relations fécondes entre ces deux arts. Je retiendrais finalement tous les écrits sur la théorie de l'essai, pour leur contribution essentielle à notre connaissance du genre, et même le dernier, sur Lucièn Goldmann, pour la leçon de rigueur et de cohérence qui est servie à ce dernier.

Ce choix est sans exclusive, on l'aura compris. De fait, outre les qualités déjà signalées de cet écrivain, il est une démarche ou une méthode chez lui qui rend profitable la lecture de chacun des textes. Son recours fréquent à ce qu'il appelle l'anthropologique ou l'anthropologie culturelle, afin de comprendre un phénomène ou une œuvre, donne une épaisseur de conscience à ce qui autrement risquerait d'être in-signifiant. Le recours aux "tendances lourdes de l'histoire" (p. 230) enracine le phénomène dans des raisons profondes, fondamentales, liées à la condition humaine et à la permanence de certains comportements. L'analyse fait alors jaillir une lumière qui quelquefois étonne et le plus souvent entraîne l'adhésion.

En somme, je sors de cette lecture plus convaincu que jamais que les études médiévales et classiques sont une des voies privilégiées d'accès nón seulement à la littérature (y compris la québécoise), mais aussi à la modernité. 


\section{À livre ouvert}

Le livre de Laurent Mailhot est à la fois semblable et très différent. Il est classique par sa présentation: les sections sur "Le livre", *La poésie -, "Le roman ", "L'essai ", :Le théâtre " et "Autres regards * (c'est-àdire, le regard des Autres sur la littérature québécoise), présentent un certain nombre d'études sur la littérature québécoise, études qui doninent une place de choix au parcours, au survol, aux présentations d'ensemble, très souvent pour auditeurs ou lecteurs étrangers (Américains, Français, Italiens, Belges, Canadians). Laurent Mailhot, d'ailleurs, excelle dans les bilans et les rétrospectives. Il a l'art de fixer en quelques traits rapides un écrivain, une ouvre, une évolution. Cela est cursif, incisif et d'une grande érudition. Le lecteur trouvera donc dans ce recueil plusieurs aide-mémoire exemplaires et précieux, soịt sur la bibliothèque idéale québécoise ("Classiques canadiens"), soit sur le roman ("Le roman québécois et ses langages"), l'essai ("L'écriture de l'essai "), le théâtre (*D'une histoire dramatique à un espace théâtral "). Il trouvera également plusieurs études dont l'objet est plus limité: les plus percutantes me semblent celles sur les poètes (Émile Nelligan, Jacques Brault, Gilles Hénault) ou sur un poète prosateur (Fernand Ouellette). Ces deux dernières études, en particulier ("Signaux et signes de Gilles Hénault ", "Récit/essai: le Journal dénoué de Fernand Ouellette•), m'apparaissent d'une remarquable densité et d'une grande richesse. Chaque mot porte: le critique semble vibrer au diapason de son sujet, qu'il éclaire de l'intérieur. Par ailleurs, les admirateurs de Michel Tremblay apprécieront l'étude qui lui est consacrée et qui dégage le théâtre dans l'œuvre romanesque; les inconditionnels d'Yves Thériault, toutefois, trouveront certainement que le jugement porté sur ce conteur prolifique est plutôt dur. Le titre retenu pour cette étude ("La montagne et les souris chez Thériault ") annonce bien ses couleurs.

Voilà pour une brève indication du contenu du recueil, dont l'apparence traditionnelle, toutefois, camoufle une démarche particulière, personnelle et originale. En effet, on ne peut dire que Laurent Mailhot analyse ou étudie banalement une œuvre. Il accompagne, il traverse, il commente, il réinvente le texte en faisant jouer, en laissant jouer, en lui, sous sa plume, les résonances du texte qu'il a sous les yeux. L'expression qu'il emprunte à Ricardou ("explorer le langage comme un espace particulier - [p. 97]) pour décrire la démarche du poète Gilles Hénault, pourrait aussi servir à décrire sa propre méthode. Le langage poétique, romanesque, théâtral, essayistique, est un espace qu'il explore, quelquefois à tâtons mais le plus souvent à grandes enjambées, en regardant ou en sondant, selon le cas, toujours en expédition de 
reconnaissance. Tout comme Gilles Hénault, pour voir l'univers, doit en faire l'équivalent d'un poème, Laurent Mailhot, pour comprendre l'œuvre, doit la refaire, la recouler dans son creuset, la traverser de ses regards ou de ses pas, la réinventer et la mettre en rapport. Peut-être surtout la mettre en rapport. Avec lui-même, mais avec d'autres œuvres aussi. Le Journal dénoué de Fernand Ouellette, par exemple, sera ainsi mis en rapport avec des entreprises analogues de Green, de Mauriac, de Kierkegaard, de Pascal, d'Omar Khazzan, de saint Augustin, de Tolstoï, d'Albert Cohen. Ce ne sont pas de banales comparaisons qu'effectue le critique. On sait, on sent que chacun de ces noms est plus qu'un nom: une vie, une œuvre, un destin. Des destins qui deviennent alors autant de repères pour baliser la route qu'est la lecture critique authentique d'une œuvre québécoise. *Toute critique est une aventure paradoxale, contradictoire: un point de départ sans arrivée définitive, un parcours qui jamais ne se répète. (p. 207), dira Laurent Mailhot. Nous sommes conviés à le suivre dans son parcours aventureux. On pense à l'homme en marche d'Ernest Gagnon, épris de liberté et de grands espaces. L'espace, en effet, est une notion fondamentale pour Laurent Mailhot. Davantage, peut-être: une fascination.

Son travail exploratoire produit un livre touffu, tout en incises, parenthèses, nuances, allusions, digressions. Une écriture rapaillante à l'extrême. Rapaillante ou rayonnante? On hésite. Est-ce qu'elle cherche à ramasser, à rassembler, à tout faire converger vers l'œuvre lue? Ne rayonne-t-elle pas plutôt, multipliant les aperçus, les éclairages, les flasb à partir du sujet? En réalité, rapaillante et rayonnante, en alternances rapides. Elle exige donc beaucoup de son lecteur: culture profonde, sensibilité à fleur de peau, attention de tous les instants, car la route aventureuse que choisit le critique est la plus ardue, hors des sentiers battus. Voici un premier de cordée qui bondit de saillie en saillie et attend de son lecteur qu'il en fasse autant.

On peut dire que cette perception d'un style et d'une conduite est un effet du recueil. À la lecture des études isolées de Laurent Mailhot, au hasard des publications, cela, du moins, ne m'avait guère frappé. Certes, il y avait un ton, un souffle et une démarche, davantage portée sur l'ellipse et l'allusion que sur la démonstration. Mais du rassemblement des écrits jaillit l'évidence. Il y a là un rythme, une écriture. Elle s'oppose à celle de Jean Marcel, plus classique. La phrase de ce demier se développe et se déploie, principale et subordonnées bien ordonnées, architexture savante, héritière d'une longue tradition thétorique. Celle de Laurent Mailhot est d'un autre rythme, syncopée, hachurée, courte, volontiers elliptique. Ou si elle s'allonge, c'est par adjonction, énumération, notations successivẹs, chaînes d'oppositions ou de synonymes, 
chaque touche ajoutant un reflet, une nuance. Elle répugne à la subordonnée, sauf la relative. Des phrases typiques seraient les suivantes:

Le livre est comme un objet magique, un coffre mystérieux, une île au trésor, une caverne d'Ali Baba: voler (changer) un titre, c'est déplacer une richesse, violer un secret, voiler une passion. (p. 33)

Coupés de leur contexte, détournés de leur fonction, déplacés, exilés, réinsérés et assimilés, ces signes, ces signaux questionnent le livre, la langue, les histoires (p. 39-40).

Par groupes de trois ou de quatre, sinon davantage, les notations s'accumulent, s'entassent, se juxtaposent, et construisent progressivement le sens. Tachisme textuel ou jazz critique, comme on le voudra, selon ses références propres.

Si je le signale, ce n'est pas pour le plaisir d'une rapide et maladroite analyse stylistique. L'auteur en serait peut-être, d'ailleurs, le premier étonné, lui qui, dans son Avant-propos, dit clairement: "Les textes ici retenus ne sont pas d'un écrivain ou d'un artiste, mais d'un écrivant, d'un artisan. [...] Disons d'un lecteur professionnel.. (p. 12) Certes, nous ne sommes pas obligés de le croire. Je serais, quant à moi, porté à mettre sur le compte de l'écriture de cet artisan tout ce qu'il dit de celle de Fernand Ouellette.

L'essai, comme la poésie et au contraire de la science, est - un savoir sans pouvoir , un savoir-faire, un acte risqué. [...] L'essai n'est jamais plein, jamais simple, jamais pur. Il est noueux, protubérant, rythmé, marqué, usé par d'incessants passages. (p. 211)

Mais, quoi qu'il en soit du crédit accordé aux protestations du préfacier, je crois qu'il importe de savoir, nous lecteurs, que ce lecteur professionnel lit à livre ouvert. Comme on dirait à tombeau ouvert. C'est-à-dire sans ménagement, sans peur des risques, prenant, au besoin, les virages sur le chapeau des roues, fonçant à toute allure, vibrant au rythme de la machine textuelle, irrespectueux de toutes les polices qui voudraient gouverner le sens et enlever au lecteur, non seulement le volant mais la clef de contact. Il lit à livre ouvert, aussi, comme on utilisait autrefois l'expression pour traduire notre émerveillement devant le professeur ou le grand cousin latiniste, pour décrire celui qui lisait couramment des textes difficiles, parce qu'il était savant et qu'il n'avait pas besoin d'avoir constamment recours à des références externes ou aux explications du maître. Il était son propre dictionnaire et sa propre référence, capable de décrypter seul les mystères de l'imprimé. À livre ouvert comme à cœur ouvert, également, sans doute. À cœur ouvert comme les amis face à face, sans subterfuges, et à cour ouvert comme le chirurgien, sorcier de la vie palpitante. La lecture est une aventure et un risque. 
Réflexions qui viennent à lire ces études mais d'abord à soupeser le titre qui les coiffe. Titre merveilleux, aux résonances multiples, qui se répercutent volontiers dans notre esprit. Mais on s'aperçoit, en commençant la lecture du recueil, dès le premier essai éponyme, que l'auteur les a toutes prévues et répertoriées, ces résonances, et même les jeux de mots possibles sur les syntagmes apparentés. "À livre ouvert, comme à tombeau ouvert et à cour ouvert. Entre la vie et la mort. Un livre se dévore comme du pain (se coupe, se tranche), se parcourt comme une route, une province, un pays.- (p. 18) Le programme de lecture était contenu dans le titre, et le livre n'y faut pas. Voici des études qui ouvrent le livre, dans tous les sens qu'on voudra donner au verbe. Et qui ont prévu, voire programmé, notre lecture.

Mais par un curieux paradoxe, comme pour ancrer ce qui peut sembler savante improvisation, endiguer son flot ou contrôler son apparente dérive, se présente tout le système des notes. Vingt, trente, quarante, voire soixante-dix notes par étude! Trente-sept pages de notes! Toutes ces réflexions, tous ces aperçus, toutes ces prises, fruits d'une chasse ou d'une battue en règle, ne sont pas gratuites, peu s'en faut. Chacune est l'effet d'une recherche, fondée et justifiée par l'appareillage critique. Les notes se lisent comme un texte second, savant et universitaire, qui fait contrepoids; se produit alors une espèce de tension féconde entre l'universitaire et le jazzman, entre l'érudit et le peintre tachiste, entre le savant et l'aventurier. Ce qui me permet de conclure en disant de ce livre qu'il est à la fois personnel et savant, emporté et érudit, fantaisiste et rigoureux, riche et dense, sans concessions dans ses exigences face aux lecteurs que nous sommes.

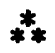

Il ne me semble pas fortuit que chacun de ces auteurs parle, dans la présentation de son recueil, d'une chasse. Le recueil est à la fois - cursus et corpus, excursion dans l'espace et incursion dans le temps, chasse et retour de la chasse ", dira Laurent Mailhot. Quant à Jean Marcel, il décèlera dans ses écrits rassemblés une trace de "l'affût de toutes [ses] chasses: la quête d'un peu de sens .

La chasse, c'est-à-dire l'attente ou la course, la patience ou la ruse, la battue ou le pistage. Le travail critique, en effet, est chasse royale, poursuite d'un gibier qui se laisse prendre mais pour aussitôt s'évanouir. Toujours pris, toujours insaisissable. La littérature. 
1. Jean Marcel, Pensées, passions et proses, Montréal, L'Hexagone, coll. -Essais littéraires , 1992, $399 \mathrm{p}$.

2. Laurent Mailhot, Ouvrir le liere, Montréal, L'Hexagone, coll. eEssais littéraires", 1992, 353 p.

\section{Parlez-vous littératuré?}

\section{Sylvain Simard, Université d'Ottawa}

«[Tout] est accroché à cet élément essentiel, en découle ou nous y ramène infailliblement. (René Lévesque, Option Québec, p. 19). Cet "élément essentiel", c'est, bien sûr, la langue; et dans "tout ce qui nous y ramène ", il y a la littérature, et ce depuis sa naissance au XIX ${ }^{c}$ siècle. Aussi, pour mieux connaître celle-ci, importe-t-il d'étudier non seulement l'état de la langue, mais aussi l'évolution des perceptions qu'en ont eues écrivains et critiques au Québec et en France.

L'auteure de Langue et Littérature au Québec, 1895-1914¹ appartient à cette équipe du Centre de recherche en littérature québécoise (CRELIQ) de l'Université Laval dont les nombreux travaux de qualité font beaucoup pour l'avancement de la connaissance sur la littérature d'ici. Cette spécialiste de l'œuvre critique de Charles ab der Halden privilégie une approche sociocritique inspirée surtout des travaux théoriques de Pierre Bourdieu et de ses collaborateurs des Actes de la recherche en sciences sociales. Inversant les termes habituels des rapports entre la langue et la littérature, Marie-Andrée Beaudet, dans cet essai fouillé, brillant et limpide, expose l'influence de la situation linguistique sur la formation d'un champ littéraire au Québec.

C'est d'ailleurs cette notion de "champ littéraire", plutôt que celle d'* institution littéraire, notion moins dynamique puisqu'elle implique une antériorité de la constitution et un certain déterminisme sur les "constituants", qu'adopte d'emblée l'auteur. Après avoir résumé de façon succinte le cadre linguistique général prévalant au Canada français, elle s'approprie, pour y donner suite, certaines hypothèses d'André Belleau sur le romancier québécois qui vit sa culture * dans la distance, comme le fait d'une autre classe, d'un autre pays [...]. et pour qui la situation linguistique concrète de l'écrivain québécois * nous oblige à poser le problème de la norme même de la langue littéraire [...]* à titre 